\title{
Design of a bent beam electrothermal actuator for in situ tensile testing of ceramic nanostructures
}

\author{
Maria F. Pantano ${ }^{1}$, Nicola Pugno ${ }^{1,2,3 *}$ \\ ${ }^{1}$ Laboratory of Bio-inspired \& Graphene Nanomechanics, Department of Civil, Environmental and Mechanical \\ Engineering, University of Trento, Via Mesiano 77, 38123 Trento, Italy \\ ${ }^{2}$ Center for Materials and Microsystems, Fondazione Bruno Kessler, Via Sommarive 18, 38123 Povo (TN), Italy \\ ${ }^{3}$ School of Engineering and Materials Science, Queen Mary University of London, Mile End Road, London E1 4NS, U.K. \\ *Corresponding author: nicola.pugno@ unitn.it; Tel.: +390461282525
}

\begin{abstract}
A necessary condition to include nanoscale materials in the design of highly performing as well as reliable electrical and electromechanical devices is the availability of a sufficiently deep knowledge of their mechanical behavior. Up to date, the most powerful tools for mechanical characterization of nanosamples are properly designed microelectromechanical systems (MEMS), due to their compatibility with Scanning/Transmission Electron Microscopy (SEM/TEM) and high resolution force/displacement measurements. Herein, we report about the design of a MEMS platform for in situ SEM tensile testing of nanoscale samples. This is characterized by a very compact structure, based only on a bent beam electrothermal actuator, which performs both actuating and sensing functions. The size of the structural components of the present device is chosen with the aim of testing ceramic nanowires, but the resulting configuration can be applied also for other material samples.
\end{abstract}

Keywords: MEMS; mechanical testing; SEM/TEM; ceramics; nanowires.

\section{Introduction}

The outstanding properties reported for nanoscale materials, like nanosheets, nanowires and nanotubes, can be exploited in a new generation of high-performing electrical and electromechanical devices [1-2]. In this context, an exemplary case is given by graphene which presents excellent mechanical, electrical and optical properties (e.g., Young modulus and carrier mobility, to cite only a few) [3-4]. Furthermore, attention has also been devoted to different kinds of nanowires, like silicon nanowires, characterized by giant piezoresistance effects [5], as well as ceramic nanowires, like zinc oxide and gallium nitride nanowires [6-7], whose piezoelectric properties make them suitable for energy harvesting applications in self-powered nanodevices.

A key factor enabling for an accurate and reliable design with such new materials is the availability of sufficient information about their mechanical, as well as electromechanical, behavior [8]. However, in spite of the intense work already done, many questions about the behavior of materials at the micro- and nanoscale remain still open. The scattering or lack of experimental data, as well as the need of validity assessment, testify that there is still room for further investigation [9]. This is a challenging task, though.

In fact, the small size characterizing nanoscale structures compromises the effectiveness of manipulation and testing with traditional techniques. For this reason, during the years, suitable experimental protocols and testing systems have been developed. Among these, the most promising techniques are based on Micro-ElectroMechanical Systems (MEMS) technology. The most important advantage they offer relies on the compatibility of MEMS devices with Scanning/Transmission Electron Microscope (SEM/TEM) chambers, which enable for real-time imaging of the sample deformation, while providing high displacement and force resolution [10]. 
This is the post-print version of the article M.F. Pantano, N.M. Pugno. Design of a bent beam electrothermal actuator for in situ tensile testing of ceramic nanostructures. Journal of the European Ceramic Society. 34(11): 2767-2773, 2014.

The Publisher's version is available at http://dx.doi.org/10.1016/j.jeurceramsoc.2013.12.001

The literature offers different examples of MEMS-based tensile testing stages. Most of them share the same architecture, composed of an actuator (which applies force/displacement to a sample) and a sensor (for force/displacement measurement) with a small gap in between for sample positioning [11-13]. While the actuator may be thermal [11] or electrostatic [13], the sensor may be mechanical [12-13] or electrical [11]. In the first case, the sensor deformation during the test is acquired through SEM pictures, and then converted into force by multiplying it by the sensor spring constant [13] whereas, in the second case, more sophisticated design and experimental setup are required in order to provide an electrical measurement of the sensor deformation (e.g., load) [11]. In this kind of architecture, the sensor and the sample experience the same load. However, the literature offers other examples, where the tensile stage is arranged in order for the sensor and the sample to undergo instead the same displacement. Some examples can be found in [14-15], where the thermal/electrostatic actuator performs both actuating and sensing functions. Into another case [16], the displacement is applied through a nanoindenter which pushes against a MEMS platform, where a system of springs convert the incoming compressive action into a tensile force delivered to the sample.

The tensile stage presented in this paper has a quite simple structure, only comprising a bent beam motor. This has been classically used as actuator [17], but in the present device it serves also as load sensor. Details about its design will be diffusively discussed in the following sections. In particular, the size of its structural components is chosen with the aim of testing ceramic samples, like zinc oxide and gallium nitride nanowires. Nevertheless, the resulting configuration can be applied for other material samples.

\section{Device presentation and design requirements}

The proposed MEMS stage is basically composed of a bent beam motor, as depicted in figure 1. With respect to a previous proposed device [18], in this case there is no need for any additional sensing structures, since the motor can be designed in order to perform both actuation and sensing functions, as will be explained in the following.

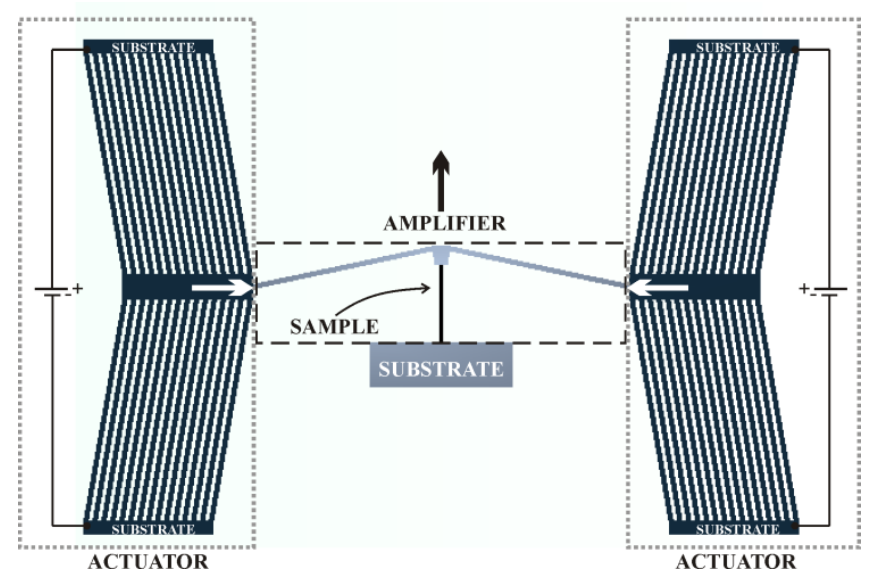

Figure 1: Schematic of the proposed tensile testing stage, where the sample is connected to a bent beam motor at one end, and anchored to the substrate at the other end.

From a structural point of view, the device includes two thermal actuators (placed at the right and left side in figure 1) provided with a classical v-shaped beams configuration. When a voltage is applied between their anchor points, current flows and generates heat by Joule effect. Thus, the beams expand, moving horizontally along the direction indicated by the arrows in figure 1 . Such displacement is delivered on each side of the central 
This is the post-print version of the article M.F. Pantano, N.M. Pugno. Design of a bent beam electrothermal actuator for in situ tensile testing of ceramic nanostructures. Journal of the European Ceramic Society. 34(11): 2767-2773, 2014.

The Publisher's version is available at http://dx.doi.org/10.1016/i.jeurceramsoc.2013.12.001

structure (e.g., referred to as amplifier in figure 1). This comprises a number of v-shaped beams, whose central part is connected to a shuttle, which is in turn connected to the sample to be tested (anchored to the substrate on the other side). The most interesting feature of the amplifier is concerned with its vertical displacement (which is also transferred to the sample) which results from the horizontal movements of the side actuators. In fact, upon proper design, the magnitude of such vertical displacement can be few times greater than the original horizontal displacement.

The novelty of the present configuration is, however, the sensing function, rather than displacement magnification, intended to be performed by the amplifier. This can be achieved by providing the amplifier with a stiffness comparable to that of the sample to be tested. In this way, the presence of the sample affects the vertical displacement achieved by the amplifier into a relatively significant amount. Thus, the difference between the displacement with and without a sample mounted can then be converted into force, by multiplying it by the amplifier stiffness. A similar idea was at the basis of the thermal actuator/sensor considered in [14]. However, in that case there was a significant temperature gradient affecting the sample. In this case, as in [18], the temperature increase due to the Joule effect is not a problem, since the actuators can operate at low voltage (e.g., low temperature), thus generating a small displacement, because this is then amplified by the central structure.

The performance the device has to achieve depends on the material sample to be tested. Because of the increasing attention gained by ceramic nanowires, the present design is customized with reference to exemplary zinc oxide/ gallium nitride nanowires. In particular, considering nanowires with $4 \mu \mathrm{m}$ length and diameters up to $100 \mathrm{~nm}$, and given their mechanical properties [19-21], the device should be able to generate up to $1 \mu \mathrm{m}$ displacements and force up to 100-200 $\mu$ N. However, the device is versatile and other nanostructures than these could be tested, too.

The following sections provide more details about the design methodologies of the thermal actuators and mechanical amplifier, including both analytical and mutiphysics numerical modeling.

\section{Design of the thermal actuators}

The thermal actuators have a classical configuration with a freestanding shuttle, anchored to the substrate through a series of v-shaped beams. When a voltage is applied across the v-shaped beams, the corresponding current flow generates heat by Joule effect. The dissipated heat causes thermal expansion of the beams, which results into a horizontal movement (figure $2 \mathrm{a}$ ).

According to a detailed analysis reported in [22], the actuator axial stiffness and displacement can be expressed as:

$$
u=\alpha \Delta T \frac{\sin \theta}{\sin ^{2} \theta+\left(\frac{b}{L}\right)^{2} \cos ^{2} \theta}
$$

and

$K=2 n\left(\frac{E A}{L} \sin ^{2} \theta+\frac{12 E I}{L^{3}} \cos ^{2} \theta\right)$

where $\Delta T$ is the temperature gradient, $E$ is the Young modulus, $I$ is the moment of inertia, $A$ is the transversal area, $b$ is the width, $L$ is the length, $t$ is the thickness, $\theta$ is the inclination angle with respect to the vertical axis, and $n$ is the number of v-shaped beams. 
This is the post-print version of the article M.F. Pantano, N.M. Pugno. Design of a bent beam electrothermal actuator for in situ tensile testing of ceramic nanostructures. Journal of the European Ceramic Society. 34(11): 2767-2773, 2014.

The Publisher's version is available at http://dx.doi.org/10.1016/j.jeurceramsoc.2013.12.001
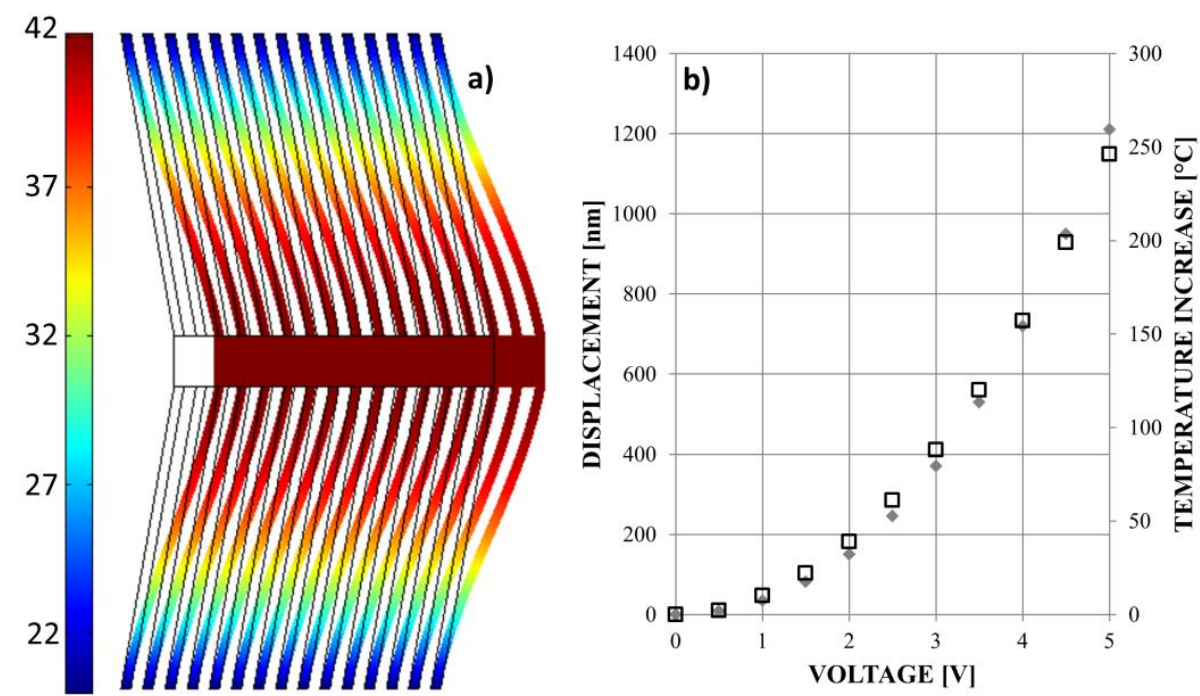

Figure 2: a) Temperature field $\left[{ }^{\circ} \mathrm{C}\right]$ over the thermal actuator biased with $1.5 \mathrm{~V}$; b) The delivered displacement (grey diamond) and temperature (hollow squares) on the actuator as functions of the bias voltage.

The geometry of the actuator has to be chosen in order for its stiffness to be significantly higher than the expected stiffness of the sample (thus guaranteeing its displacement to not be affected by the sample).

Figure 2 shows the maximum horizontal displacement and temperature of either thermal actuator, included in the device, as functions of the bias voltage. These results were obtained from a numerical multiphysics analysis, performed with Comsol Multiphysics, considering 15 polysilicon $250 \mu \mathrm{m}$ long, $6 \mu \mathrm{m}$ wide and $22 \mu \mathrm{m}$ thick vshaped beams, with inclination angle $\theta=10^{\circ}$ (the corresponding actuator stiffness is about $74400 \mathrm{~N} / \mathrm{m}$ ). For computation, the following physical parameters were considered: Young modulus, $E=152.9 \mathrm{GPa}$ [23], Poisson ratio, $v=0.2$ [23], resistivity, $\rho=3.74 \cdot 10-4 \Omega \mathrm{m}$, thermal expansion coefficient, $\alpha(T)\left[\mathrm{K}^{-1}\right]=(3.725\{1-\exp [1-$ $\left.5.88 \cdot 10-3(T-124)]\}+5.548 \cdot 10^{-4} T\right) \cdot 10^{-6}$ (where $T$ is temperature expressed in $[\mathrm{K}]$ ) [24], thermal conductivity, $k=34 \mathrm{Wm}^{-1} \mathrm{~K}^{-1}[25]$.

\section{Design of the mechanical amplifier}

The amplifier is the most interesting feature within the device. A simple and straightforward way to characterize its mechanical behavior is to start from the analysis of one inclined beam. This can be considered as provided with four degrees of freedom, two for each node (figure 3a-b).

It is convenient to first consider a local reference system $(\xi, \eta)$, rotated by $\theta$ with respect to the global reference system $(x, y)$. In the local reference system it follows that:

$\{F\}_{\xi, \eta}=[\widehat{K}]\{u\}_{\xi, \eta}$

where $\{F\}_{\xi, \eta}=\left\{H_{l \xi}, V_{I \eta}, H_{2 \xi}, V_{2 \eta}\right\}^{\mathrm{T}}$ is the force vector, $\{u\}_{\xi, \eta}=\left\{u_{1 \xi}, v_{I \eta}, u_{2 \xi}, v_{2 \eta}\right\}^{\mathrm{T}}$ is the displacement vector and $[\widehat{K}]$ is the stiffness matrix, which can be expressed as:

$[\widehat{K}]=\left[\begin{array}{cccc}E A / L & 0 & -E A / L & 0 \\ 0 & 12 E I / L^{3} & 0 & -12 E I / L^{3} \\ -E A / L & 0 & E A / L & 0 \\ 0 & -12 E I L^{3} & 0 & 12 E I / L^{3}\end{array}\right]$ 
This is the post-print version of the article M.F. Pantano, N.M. Pugno. Design of a bent beam electrothermal actuator for in situ tensile testing of ceramic nanostructures. Journal of the European Ceramic Society. 34(11): 2767-2773, 2014.

The Publisher's version is available at http://dx.doi.org/10.1016/j.jeurceramsoc.2013.12.001

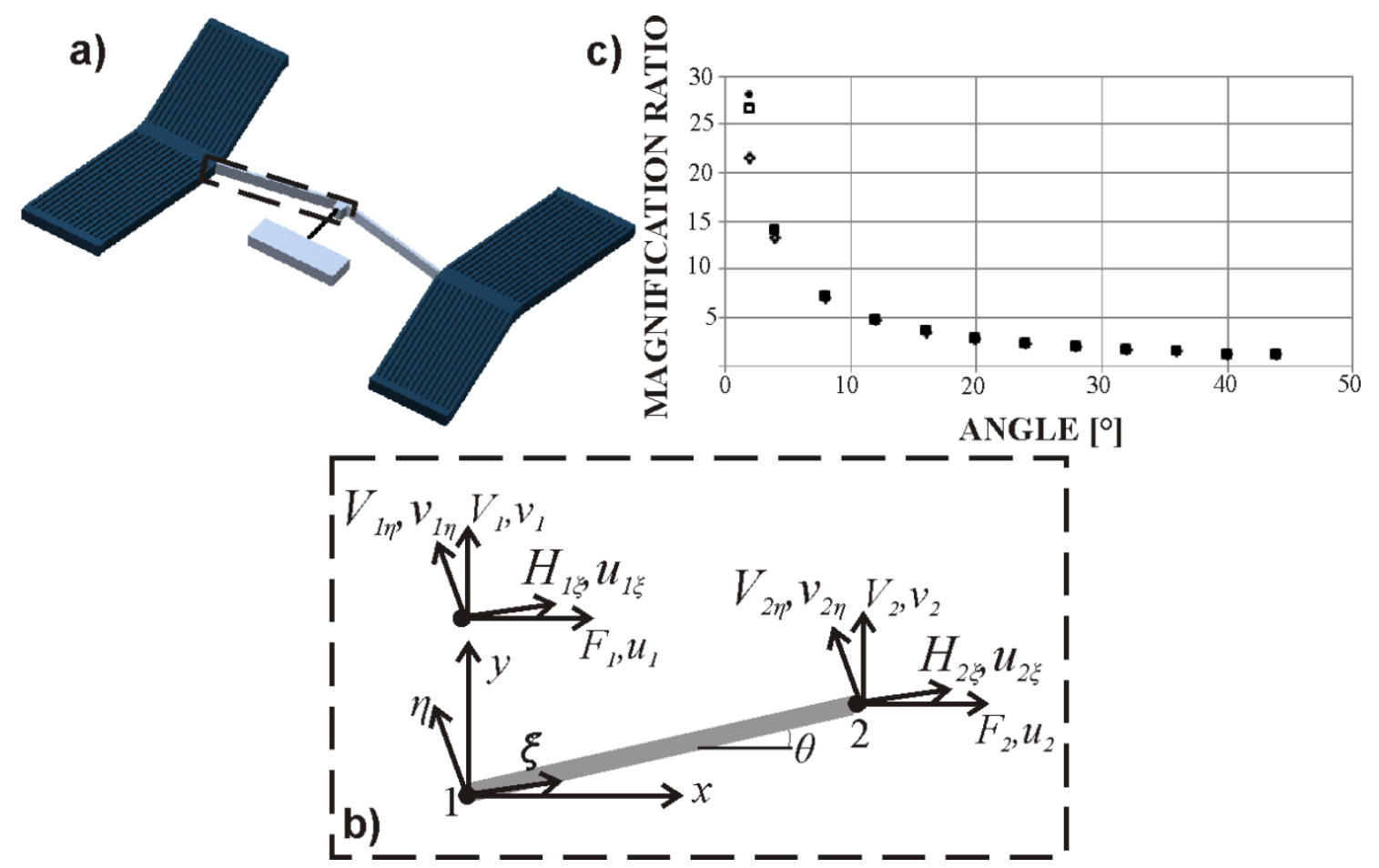

Figure 3: Reference systems for an exemplary inclined beam (b) belonging to the device amplifier (a); c) Dependence of the amplifier magnification ratio on its beams inclination angle for different values of the geometrical ratio beam width/beam length, $b / L$ (diamonds: $b / L=0.02$, rectangles $b / L=0.01$, circles: $b / L=0.005$ ).

where $E$ is the Young modulus, $A$ is the transverse area, $I$ is the moment of inertia, and $L$ is the beam length. However, the force and displacement vectors in the local reference system are related to those in the global system as $\{F\}_{\xi, \eta}=[T]\{F\}_{x, y}$ and $\{u\}_{\xi, \eta}=[T]\{u\}_{x, y}$, respectively, where $\{F\}_{x, y}=\left\{H_{l}, V_{l}, H_{2}, V_{2}\right\}^{\mathrm{T}},\{u\}_{x, y}=\left\{u_{l}, v_{l}, u_{2}\right.$, $\left.v_{2}\right\}^{\mathrm{T}}$, and

$[T]=\left[\begin{array}{cccc}\cos \theta & \sin \theta & 0 & 0 \\ -\sin \theta & \cos \theta & 0 & 0 \\ 0 & 0 & \cos \theta & \sin \theta \\ 0 & 0 & -\sin \theta & \cos \theta\end{array}\right]$

By using such relationships, equation (3) can be rewritten as:

$[T]\{F\}_{x, y}=[\widehat{K}][T]\{u\}_{x, y}$

or,

$\{F\}_{x, y}=[T]^{-1}[\widehat{K}][T]\{u\}_{x, y}$

which can be further simplified as:

$\{F\}_{x, y}=[K]\{u\}_{x, y}$ 
The Publisher's version is available at http://dx.doi.org/10.1016/j.jeurceramsoc.2013.12.001

where $[K]=[T]^{-1}[\widehat{K}][T]$ is the beam stiffness matrix in the global reference system. After some calculation, equation (8) can be written in an extended form as:

$$
\left\{\begin{array}{l}
H_{1} \\
V_{1} \\
H_{2} \\
V_{2}
\end{array}\right\}=\left[\begin{array}{cccc}
k_{11} & k_{12} & k_{13} & k_{14} \\
& k_{22} & k_{23} & k_{24} \\
S Y M M & & k_{33} & k_{34} \\
& & & k_{44}
\end{array}\right]\left\{\begin{array}{l}
u_{1} \\
v_{1} \\
u_{2} \\
v_{2}
\end{array}\right\}
$$

Where:

$$
\begin{aligned}
& k_{11}=\frac{E A}{L} \cos ^{2} \theta+12 \frac{E I}{L^{3}} \sin ^{2} \theta \\
& k_{12}=\frac{E A}{L} \cos \theta \sin \theta-12 \frac{E I}{L^{3}} \cos \theta \sin \theta \\
& k_{13}=-\frac{E A}{L} \cos ^{2} \theta-12 \frac{E I}{L^{3}} \sin ^{2} \theta \\
& k_{14}=-\frac{E A}{L} \cos \theta \sin \theta+12 \frac{E I}{L^{3}} \cos \theta \sin \theta \\
& k_{22}=\frac{E A}{L} \sin ^{2} \theta+12 \frac{E I}{L^{3}} \cos ^{2} \theta \\
& k_{23}=-\frac{E A}{L} \cos \theta \sin \theta+12 \frac{E I}{L^{3}} \cos \theta \sin \theta \\
& k_{24}=-\frac{E A}{L} \sin { }^{2} \theta-12 \frac{E I}{L^{3}} \cos ^{2} \theta \\
& k_{33}=\frac{E A}{L} \cos { }^{2} \theta+12 \frac{E I}{L^{3}} \sin ^{2} \theta \\
& k_{34}=\frac{E A}{L} \cos \theta \sin \theta-12 \frac{E I}{L^{3}} \cos \theta \sin \theta \\
& k_{44}=\frac{E A}{L} \sin ^{2} \theta+12 \frac{E I}{L^{3}} \cos ^{2} \theta=k_{A}
\end{aligned}
$$

This latter parameter has also an important physical meaning, being the beam stiffness along the $y$-axis $\left(k_{A}\right)$.

Because of symmetry, no horizontal movement is allowed to node 2 (figure $3 \mathrm{a}-\mathrm{b}$ ), which implies that $u_{2}=0$. Furthermore, without any sample mounted, $v_{l}=0$ and $V_{2}=0$. In this condition, the fourth row of matrix equation (9) provide a relationship between the horizontal displacement $u_{1}$ (due to the side thermal actuator) and the corresponding vertical displacement $v_{2}$ :

$k_{41} u_{1}+k_{44} v_{2}=0$

or simply

$$
v_{2}=-\frac{k_{41}}{k_{A}} u_{1}
$$

Combining relationships (10) with equations (11) and (12), these latter become: 
This is the post-print version of the article M.F. Pantano, N.M. Pugno. Design of a bent beam electrothermal actuator for in situ tensile testing of ceramic nanostructures. Journal of the European Ceramic Society. 34(11): 2767-2773, 2014.

The Publisher's version is available at http://dx.doi.org/10.1016/j.jeurceramsoc.2013.12.001

$\left(-\frac{E A}{L} \cos \theta \sin \theta+12 \frac{E I}{L^{3}} \cos \theta \sin \theta\right) u_{1}+\left(\frac{E A}{L} \sin ^{2} \theta+12 \frac{E I}{L^{3}} \cos ^{2} \theta\right) v_{2}=0$

and

$v_{2}=\frac{\left(1-\left(\frac{b}{L}\right)^{2}\right) \cos \theta \sin \theta}{\sin ^{2} \theta+\left(\frac{b}{L}\right)^{2} \cos ^{2} \theta} u_{1}$

respectively. Equation (14) shows that $v_{2}$ is proportional to $u_{l}$, with the proportionality constant depending only on the geometrical ratio beam width/ beam length $(b / L)$ (figure $3 \mathrm{c})$. It results that the magnification ratio $\left(v_{2} / u_{1}\right)$ increases as the beam inclination angle and the ratio $b / L$ decrease. In particular, for $\theta<10^{\circ}$ the ratio $b / L$ has a significant influence on the magnification ratio, which can be even much bigger than 10 .

When a sample is mounted onto the device, the force acting on the amplifier $\left(V_{2} \neq 0\right)$ is half of the same needed to deform the sample. Still referring to the fourth row of matrix equation (9) and assuming $v_{l}=0$ (e.g., the vertical displacement on the actuator side is negligible, which is true if this is sufficiently stiff), it follows that:

$k_{41} u_{1}+k_{A} v_{2}=V_{2}$

With reference to eq. (12), eq. (15) can be rewritten as:

$k_{A}\left(v_{20}-v_{2}\right)=V_{2}$

where $v_{20}$ is the amplifier displacement, when no sample is present. In the sample linear regime, $V_{2}=k_{s} v_{2} / 2$, with $k_{s}$ being the sample stiffness, and eq. (16) can be rearranged as:

$v_{2}=\frac{2 k_{A}}{k_{S}+2 k_{A}} v_{20}$

Thus, in order for the amplifier displacement $v_{2}$ to be significantly affected by the sample presence, it is necessary for $k_{A}$ to be comparable with $k_{s}$. In this condition, the amplifier can be efficiently used as load sensor, with the load being measured from the displacement difference produced by the sample presence (e.g., $\left.v_{20}-v_{2}\right)$.

From the equation (15), it is possible to evaluate also the maximum load which the amplifier can withstand (e.g., the load preventing any displacement $v_{2}$ ) as:

$V_{\max }=k_{41} u_{1}$

This is also half of the maximum load which can be applied to the sample.

Referring to ceramic (like zinc oxide/gallium nitride) nanowires, with length of $4 \mu \mathrm{m}$ and diameter not bigger than $100 \mathrm{~nm}$, a good amplifier is expected to have a stiffness of around $300 \mathrm{~N} / \mathrm{m}$ and should be able to apply $100-200 \mu \mathrm{N}$. If polysilicon is considered as structural material, an amplifier with a pair of $250 \mu \mathrm{m}$ long, $6 \mu \mathrm{m}$ 
This is the post-print version of the article M.F. Pantano, N.M. Pugno. Design of a bent beam electrothermal actuator for in situ tensile testing of ceramic nanostructures. Journal of the European Ceramic Society. 34(11): 2767-2773, 2014.

The Publisher's version is available at http://dx.doi.org/10.1016/i.jeurceramsoc.2013.12.001

wide, and $22 \mu \mathrm{m}$ thick beams ${ }^{1}$, with inclination angle $\theta=2^{\circ}$ fulfills both requirements. In fact, according to the last of expressions (10), $k_{A}=289 \mathrm{~N} / \mathrm{m}$, while according to equation (14), such geometry allows to achieve an amplification ratio (without any sample) $v_{2} / u_{1}=19.44$. In particular, it is interesting to note that with this amplification ratio, it is possible to achieve a sample displacement bigger than $1 \mu \mathrm{m}$ (one of the design constraints) with a small actuator displacement $(\sim 60 \mathrm{~nm})$, and thus a small temperature increase $\left(\sim 20^{\circ} \mathrm{C}\right.$ from figure $2 b$ ), while being able to apply forces bigger than $200 \mu \mathrm{N}$.

In order to assess the validity of the analytical model, a set of numerical analyses was performed. For the geometry chosen during the present design, the numerical amplification ratio differed from the analytical expectation by less than $1 \%$, thus testifying the reliability of the analytical procedure.

\section{Discussion about the design results}

From the analytical model derived in the previous section, it was shown that there is a linear relationship between the displacement delivered by the side thermal actuators and the resulting amplifier displacement (equation (12)). However, this linear relationship is an underestimation of the real behavior. In fact, the heat generated by the thermal actuators causes a temperature increase inside the amplifier beams (figure 4a). Thus, these tend to expand causing an additional vertical displacement to be summed to the displacement due to the actuators movement.

a)

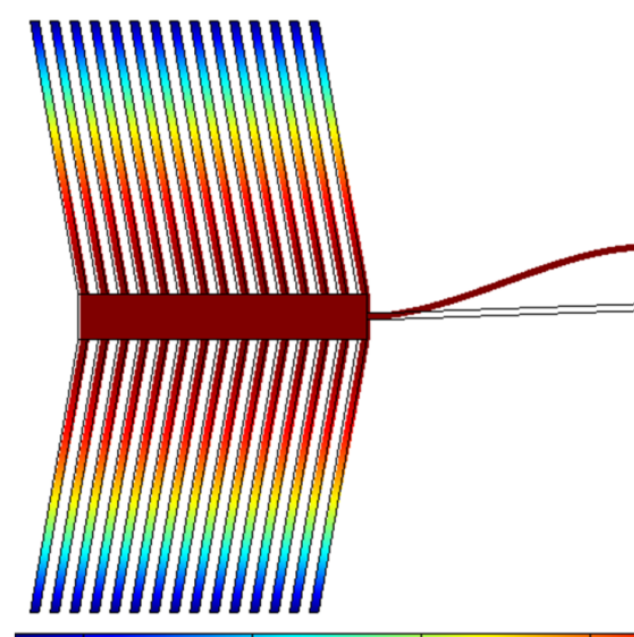

22

\begin{abstract}
27
\end{abstract}

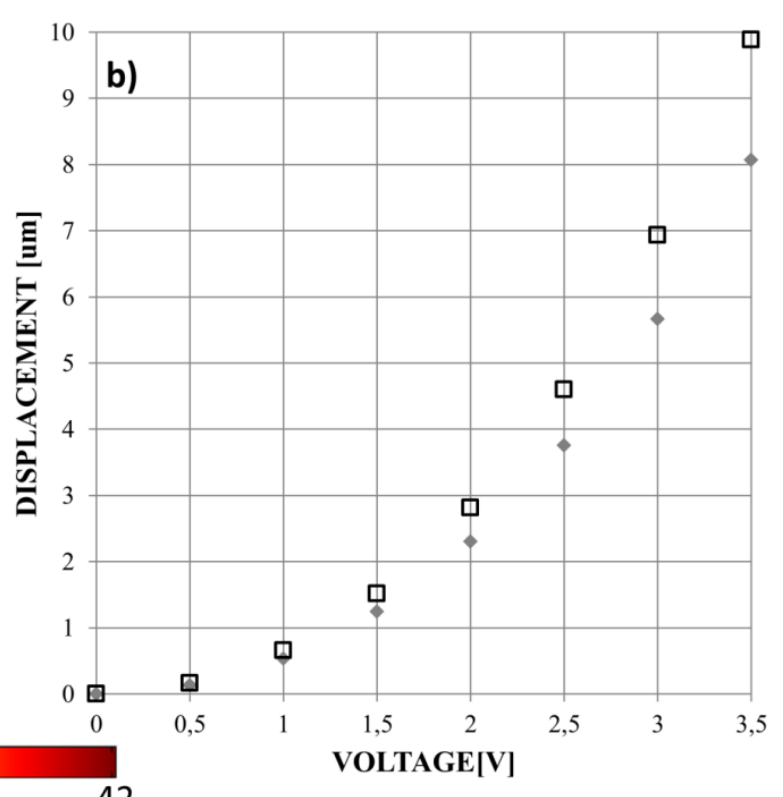

42

Figure 4: a) Temperature field $\left[{ }^{\circ} \mathrm{C}\right]$ over half device, when the thermal actuator is biased with $1.5 \mathrm{~V}$; b) Relationship between the amplifier displacement and the actuator bias voltage when the amplifier thermal expansion is neglected (grey diamonds) and when this is included into the numerical analysis (hollow squares).

\footnotetext{
${ }^{1}$ For the beam structure verification, it is possible to refer to the rows of matrix equation (9), which provide an expression of the acting forces as functions of the amplifier displacement.
} 
This is the post-print version of the article M.F. Pantano, N.M. Pugno. Design of a bent beam electrothermal actuator for in situ tensile testing of ceramic nanostructures. Journal of the European Ceramic Society. 34(11): 2767-2773, 2014.

The Publisher's version is available at http://dx.doi.org/10.1016/j.jeurceramsoc.2013.12.001

As can be inferred from figure $4 \mathrm{~b}$, obtained from a multiphysics numerical analysis of half device, such additional displacement is not negligible and increases with the actuator bias voltage, as higher voltages cause higher temperature (e.g., thermal expansion) to be achieved.

Further comments can be added also on equation (16). This relates the load on the sample to the amplifier stiffness and displacement (with and without the sample). However, the stiffness of the amplifier depends on its geometry (expression (10)), and this changes during the test, as a consequence of the movement of its beams. In order to show better this effect, it is useful to refer to the results obtained from a numerical analysis (still performed through Comsol Multiphysics), where we simulated half of the device structure (like that shown in figure 4a). We biased the thermal actuator with $1.5 \mathrm{~V}$ and applied a $50 \mu \mathrm{N}$ downward load to the amplifier beam (e.g., $100 \mu \mathrm{N}$ sample force), and we recorded the corresponding horizontal displacement of the actuator and vertical displacement of the amplifier beam. We then introduced the corresponding numbers in equation (16) and compared the result to the force we applied. The force resulting from equation (16) is about $3 \%$ less than $50 \mu \mathrm{N}$. However, such evaluation can be improved if we introduce an average amplifier stiffness in equation (16). In particular, if we average the initial amplifier stiffness and that one corresponding to the deformed amplifier geometry, then the error becomes smaller than $1 \%$.

In these numerical analyses, we applied a voltage between the ends of the thermal actuators v-shaped beams, as preferred operation mode. However, there is another interesting option which can be considered, which allows for performance of combined electrical and mechanical tests. In this second case, a voltage is applied between the ends of the inclined beams and the actuator shuttle, which can be then grounded. In this way, the amplifier and the end of the sample connected to it result to be grounded, too. Thus, the sample is electrically isolated from the remaining of the device and electrical measurements can be performed while stretching it.

\section{Conclusions}

The device presented herein the paper has a simple and compact structure, consisting only of a bent beam motor. This is provided with the double task to deliver displacements to a sample and simultaneously sense the corresponding applied loads. A detailed description of the design was presented, including the development of a simple analytical model able to characterize the mechanical behavior of the device structure. The results obtained from the analytical model were then validated through numerical simulations and further discussed through performance of multiphysics analyses, which allowed to achieve a deeper insight. The device was designed with the aim of testing ceramic nanowires, even though it is versatile and also other material samples can be considered. It is suitable to perform in situ SEM electromechanical tests, delivering relatively high displacement $(>1 \mu \mathrm{m})$ and forces $(>200 \mu \mathrm{N})$ without significant temperature increase within the sample. Its experimental applications will be the aim of future work.

\section{Acknowledgements}

NMP is supported by the European Research Council (ERC StG Ideas 2011 BIHSNAM on "Bio-Inspired hierarchical super-nanomaterials", ERC PoC -2013 REPLICA2 on "Large-area replication of biological antiadhesive nanosurfaces) and European Commission under Graphene Flagship. 
This is the post-print version of the article M.F. Pantano, N.M. Pugno. Design of a bent beam electrothermal actuator for in situ tensile testing of ceramic nanostructures. Journal of the European Ceramic Society. 34(11): 2767-2773, 2014.

The Publisher's version is available at http://dx.doi.org/10.1016/j.jeurceramsoc.2013.12.001

\section{References}

[1] Ke CH, Pugno N, Peng B, Espinosa HD. Experiments and modeling of carbon nanotube-based NEMS devices. J Mech Phys Solid 2005; 53:1314-33.

[2] Lucas M, Leach AM, McDowell MT, Hundyadi SE, Gall K, Murphy CJ, Riedo E. Plastic deformation of pentagonal silver nanowires: Comparison between AFM nanoindentation and atomistic simulations. Phys Rev B 2008; 77: 245420.

[3] Lee C, Wei X, Kysar JW, Hone J. Measurement of Elastic Properties and Intrinsic Strength of Monolayer Graphene. Science 2008; 321: 385-8.

[4] Geim AK, Novoselov KS. The rise of graphene, Nature Mater 2007; 6: 183-91.

[5] He R, Yang P. Giant piezoresistance effect in silicon nanowires. Nature Nanotechnol 2006; 1: 42-6.

[6] Wang ZL, Song J. Piezoelectric Nanogenerators based on zinc oxide nanowire arrays. Science 2006; 312 : 242-6.

[7] Su WS, Chen YF, Hsiao CL, Tu LW. Generation of electricity in GaN nanorods induced by piezoelectric effect. Appl Phys Lett 2007; 90: 063110.

[8] Pugno N, Peng B, Espinosa HD. Predictions of the strength in MEMS components with defects-a novel experimental-theoretical approach. Int J Solid Struct 2005; 42: 647-61.

[9] Mc Dowell MT, Leach AM, Gall K. On the elastic modulus of metallic nanowires. Nanolett 2008; 8 (11): 3613-8.

[10] Pantano MF, Espinosa HD, Pagnotta L. Mechanical characterization of materials at small length scales. J Mech Sci Technol 2012; 26 (2): 545-61.

[11] Espinosa HD, Zhu Y, Moldovan N. Design and Operation of a MEMS-Based Material Testing System for Nanomechanical Characterization. J Microelectromech Syst 2007; 16 (5): 1219-31.

[12] Haque MA, Saif MTA. In-situ Tesnsile Testing of Nano-scale Specimens in SEM and TEM. Exp Mech 2002; 42 (1): 123-8.

[13] Zhang D, Breguet JM, Clavel R, Sivakov V, Christiansen S, Michler J. In situ Electron Microscopy Mechanical Testing of Silicon Nanowires Using Electrostatically Actuated Tensile Stages. J Microelectromech Syst 2010; 9 (3): 663-74.

[14] Brown JJ, Suk JW, Singh G, Baca AI, Dikin DA, Ruoff RS, Bright VM. Microsystem for nanofiber electromechanical measurements. Sens Actuators A 2009; 155: 1-7.

[15] Gerratt AP, Penskiy I, Bergbreiter S, In situ characterization of PDMS in SOI-MEMS, J Micromech Microeng 2013; 23: 045003.

[16] Guo H, Chen K, Oh Y, Wang K, Dejoie C, Syed Asif SA, Warren OL, Shan ZW, Wu J, Minor AM, Mechanics and Dynamics of the Strain-Induced M1-M2 Structural Phase Transition in Individual $\mathrm{VO}_{2}$ Nanowires. Nanolett 2011; 11: 3207-13.

[17] Park JS, Chu LL, Oliver AD, Gianchandani YB. Bent-Beam Electrothermal Actuators-Part II: Linear and Rotary Microengines. J Microelectromech Syst 2001; 10 (2): 255-62.

[18] Abbas K, Alaie S, Leseman ZC. Design and characterization of a low temperature gradient and large displacement thermal actuators for in situ mechanical testing of nanoscale materials. J Micromech Microeng 2012; 22: 125027.

[19] Hoffmann S, Ostlund F, Michler J, Fan HJ, Zacharias M, Christiansen SH, Ballif C. Fracture strength and Young's modulus of ZnO nanowires. Nanotechnol 2007; 18: 205503.

[20] Chen CQ, Shi Y, Zhang YS, Zhu J, Yan YJ. Size Dependence of Young's Modulus in ZnO Nanowires, Phys Rev Lett 2006; 96: 075505. 
This is the post-print version of the article M.F. Pantano, N.M. Pugno. Design of a bent beam electrothermal actuator for in situ tensile testing of ceramic nanostructures. Journal of the European Ceramic Society. 34(11): 2767-2773, 2014.

The Publisher's version is available at http://dx.doi.org/10.1016/j.jeurceramsoc.2013.12.001

[21] Bernal RA, Agrawal R, Peng B, Bertness KA, Sanford NA, Davydov AV, Espinosa HD. Effect of Growth Orientation and Diameter on the Elasticity of GaN Nanowires. A Combined in Situ TEM and Atomistic Modeling Investigation. Nanolett 2011; 11 (2): 548-55.

[22] Zhu Y, Corigliano A, Espinosa HD. A thermal actuator for nanoscale in situ microscopy testing: Design and characterization. J Micromech Microeng 2006; 16 (2): 242-53.

[23] Mariani S, Ghisi A, Corigliano A, Zerbini S. Multiscale Analysis of MEMS Sensors Subject to Drop Impacts. Sensors 2007; 7: 1817-33.

[24] Okada Y, Tokumaru Y. Precise Determination of lattice-Parameter and Thermal Expansion Coefficient of Silicon between 300-K and 1500-K. J Appl Phys 1984; 56 (2): 314-20.

[25] Chiao M, Lin L. Self-buckling of micromachined beams under resistive heating. J Microelectromech Syst 2000; 9: 146-51. 